\title{
Global assessment and mapping of changes in mesoscale landscapes: 1992-2015
}

\author{
Jakub Nowosad, Tomasz F. Stepinski*, Pawel Netzel \\ Space Informatics Lab, Department of Geography and GIS, University of Cincinnati, Cincinnati, USA, OH 45221-0131, USA
}

\begin{abstract}
Monitoring global land cover changes is important because of concerns about their impact on environment and climate. The release by the European Space Agency (ESA) of a set of worldwide annual land cover maps covering the 19922015 period makes possible a quantitative assessment of land change on the global scale. While ESA land cover mapping effort was motivated by the need to better characterize global and regional carbon cycles, the dataset may benefit a broad range of disciplines. To facilitate utilization of ESA maps for broad-scale problems in landscape ecology and environmental studies, we have constructed a GIS-based vector database of mesoscale landscapes patterns of land cover categories in $9 \mathrm{~km} \times 9 \mathrm{~km}$ tracts of land. First, we reprojected ESA maps to the Fuller projection to assure that each landscape in the database has approximately the same size and shape so the patterns of landscapes at different locations can be compared. Second, we calculated landscape attributes including its compositions in 1992 and 2015, magnitude of pattern change, categories transition matrix for detailed characterization of change, fractional abundances of plant functional types (PFTs) in 1992 and 2015, and change trend type - a simple, overall descriptor of the character of landscape change. Combining change trends and change magnitude information we constructed a global, thematic map of land change; this map offers a visualization of what, where, and to what degree has changed between 1992 and 2015. The database is SQL searchable and supports all GIS vector operations. Using change magnitude attribute we calculated that only $22 \%$ of total landmass experienced significant landscape change during the 1992-2015 period, but that change zone accounted for $80 \%$ of all pixel-based transitions. Dominant land cover transitions were forest $\rightarrow$ agriculture followed by agriculture $\rightarrow$ forest. Using PFTs attributes to calculate global aggregation of gross and net changes for major PFTs yielded results in agreement with other recent estimates.
\end{abstract}

Keywords: ESA CCI-LC dataset, mapping land change, landscapes, land cover transitions

\section{Introduction}

Land-cover change is a pervasive phenomenon caused by changing climate, and, in recent decades, by the rapid population growth and accelerated industrialization (Goldewijk et al., 2017). As a part of a positive feedback loop, land cover changes in turn directly impact climate change and environmental conditions (Bonan, 2008; Mahmood et al., 2014; Alkama and Cescatti, 2016), and have a close relationship to population migration and economic conditions (DeFries, 2013). Thus, the assessment of land-cover changes is of prime importance for the effective planning and management of resources. It provides necessary information for making

\footnotetext{
*Corresponding author

Email addresses: nowosad.jakub@gmail.com (Jakub Nowosad), stepintz@uc . edu (Tomasz F. Stepinski), pawel@netzel.pl (Pawel Netzel)
}

decisions on a trade-off between development and conservation (Vitousek et al., 1997; DeFries et al., 2004).

There is a rich literature on assessing land-cover change from remotely sensed images. For reviews see Coppin et al. (2004); Radke et al. (2005); Warner et al. (2009); Hussain et al. (2013); Lu et al. (2014), and Tewkesbury et al. (2015). From multiple conceptual and technical components which constitute a change detection method we draw attention to three: the unit of analysis (for example, a pixel or a tile), the comparison method (for example, a direct spectral comparison or a post-classification comparison) and the change type (for example, "from-to" transitions between categories of land cover or loss/gain of specific land cover type). In general, the most frequently used method uses the pixel as the unit of analysis and the from-to, postclassification change assessment method (Tewkesbury et al., 2015). However, on the global scale, due to the 
lack of temporarily consistent global thematic maps of land cover at multiple time periods, the change assessments had focused on the detection of a specific change, namely the change of the forested area (Hansen et al., 2010, 2013; Kim et al., 2014) using the pixel-based, direct spectral comparison method.

Recently, with the release by the ESA Climate Change Initiative (CCI) program of temporally consistent 1992-2015 time series of annual global land cover maps (hereafter referred to as the CCI-LC dataset), the from-to, post-classification change assessment on the global scale became feasible. Indeed, Li et al. (2018) has performed such assessment from a perspective of the utility of CCI-LC to climate change models. Specifically, they investigated whether CCI-LC is sufficient to be implemented in land surface models (LSM) to better characterize global and regional carbon cycles. Because of this focus, Li et al. (2018) aggregated CCI-LC into $0.5^{\circ} \times 0.5^{\circ}$ (a typical spatial resolution of LSMs) and translated land cover categories into plant functional types (PFTs). They performed a comparison of gross and net changes in coverages of PFTs calculated from CCI-LC with similar calculations based on other datasets (Hurtt et al., 2011; Hansen et al., 2013; Goldewijk et al., 2017; Houghton and Nassikas, 2017).

In this paper, we focus on alternative, pattern-based approach to assessing land change using the CCI-LC. This approach stems from the perspective of landscape ecology and environmental studies where frequently it is a spatial pattern of land cover categories (hereafter also referred to as a landscape) rather than categories themselves which is of interest (Wickham and Norton, 1994; Riitters, 2011; Omernik and Griffith, 2014). Therefore, we first transform CCI-LC from the raster dataset into a vector (shapefile) database of local, mesoscale landscapes (square tiles of CCI-LC pixels) which together fill the entire extent of CCI-LC. The change of local landscape during the period of $\Delta t$ is measured by a magnitude of dissimilarity between a pattern of CCI-LC categories at $t_{1}$ and a pattern of these categories at $t_{2}=t_{1}+\Delta t$ (see section 2.2). In this paper, we only consider $t_{1}=1992$ and $t_{2}=2015$ as we are interested in a net change in landscapes over the longest period of time available in the ESA dataset. Technically, our change detection can be classified as tile-based, post-classification method.

Note that pattern dissimilarity measure is rotationally and translationally invariant, so the landscape may not change even if many of its constituent pixels undergo transitions as long as the final pattern has the same composition (the shares of land cover categories) and configuration (geometric structure) as the original. Also, note that the landscape may change even if its composition remains constant as long as its before and after configurations are different. This reflects the expectation that a functioning of an ecosystem in a given landscape depends not only on its composition but also on its overall configuration, but it does not depend on the specific orientation and details of the configuration.

The pattern-based method provides three layers of information on the global land change which vary in their levels of specificity. The first is the magnitude of change of each landscape during the period of $\Delta t$. As the magnitude is a single number, this information is directly mappable on the global scale. The resultant map visualizes hot and cold spots of land change without being specific about the character of the change. We use this first layer to classify landscapes into "changed" and "unchanged."

The second layer provides the composition of CCILC categories at $t_{1}$ and $t_{2}$ for each landscape. The $t_{1} \rightarrow t_{2}$ transition matrix provides characterization of landscape change. This layer of information is analogous to what has been calculated by Li et al. (2018) but is provided at a finer spatial resolution as it is not specifically intended for LSMs . To provide checks on our results we also calculate compositions of PFTs at $t_{1}$ and $t_{2}$ for each landscape so we can compare gross and net land changes with the values published by $\mathrm{Li}$ et al. (2018).

Finally, the third layer of information is given by change trends. A change trend is a generalized single descriptor of a character of change for the entire local landscape. Every landscape is assigned one of several possible change trends which makes this information mappable on the global scale. The result is a global thematic map of change which visualizes a global spatial distribution of different land changes in a single map a compact summarization of what, where, and to what degree has changed between 1992 and 2015.

\section{Data and methods}

In this section, we describe the CCI-LC dataset and its conversion to the database of local landscapes, a method of assessing the magnitude of change by calculating dissimilarity between landscape patterns at $t_{1}$ and $t_{2}$, and the change characterization method. Fig. 1 shows a diagram outlining consecutive computational steps taken to produce a landscape change database and the global thematic map of change. 


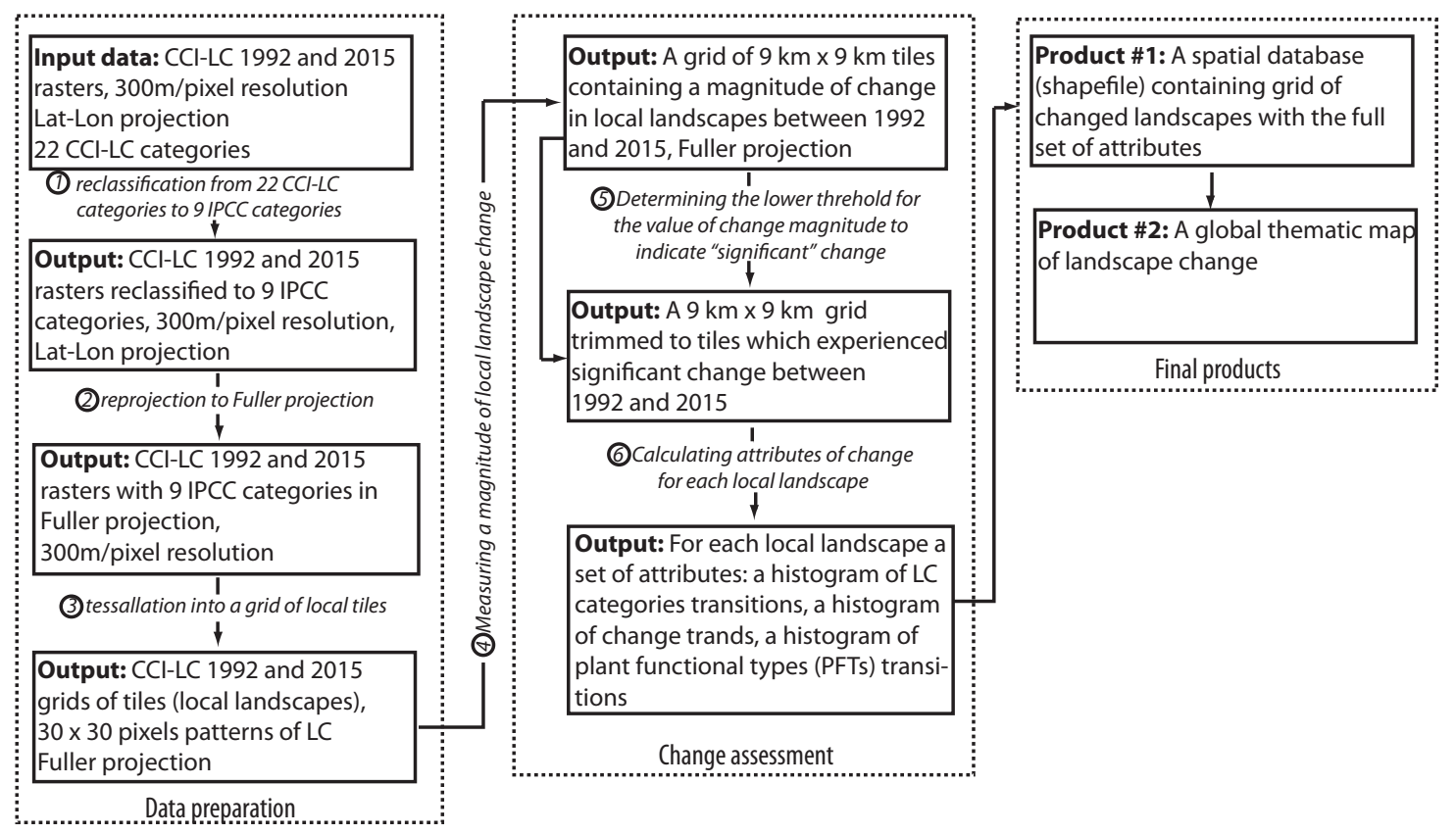

Figure 1: Diagram outlining consecutive computational steps to obtain a landscape change database. Numbers in circles label calculation steps described in the main text.

\subsection{Data}

The CCI LC maps cover 24 years from 1992 to 2015 with an annual temporal resolution and a spatial resolution of 300m (ESA, 2017). However, as we mentioned in the introduction, in this paper we only utilize 1992 and 2015 maps. Details about the CCI-LC dataset including its accuracy and the confusion table can be found in the Land Cover CCI Product User Guide V.2 (ESA, 2017); the overall accuracy of CCI-LC is estimated to be $75.4 \%$. The CCI-LC maps are in the form of $64,800 \times 129,600$ pixels Lat/Lon rasters, thus their spatial resolution is 10 arc-sec or $\sim 300 \mathrm{~m}$ at the equator.

Each pixel in CCI-LC is assigned one of 22 level 1 land cover categories which are globally consistent. There is also a level 2 legend with 37 categories which makes use of more accurate regional information where available; we do not use level 2 categories in this paper because of the lack of global consistency. Finally, CCILC categories can be grouped into 9 broader IPCC (Intergovernmental Panel on Climate Change) categories. We use IPCC categories to calculate land cover transitions characterizing landscape change (step 1 in Fig. 1). See Fig. 2 for the legend of the IPCC categories, and the CCI-LC User Guide (ESA, 2017) for correspondence between CCI-LC and IPCC categories. However, we use level $1 \mathrm{CCI}-\mathrm{LC}$ categories to compute composition of PFTs in each landscape.

Local landscapes should have all the same size and shape for their patterns to be comparable when performing SQL queries on our database. Therefore we reproject (step 2) the 1992 and 2015 maps into the Fuller projection (Gray, 1995) with $300 \mathrm{~m}$ resolution to keep distortions of tiles' real shapes and sizes below $2 \%$. Fuller-projected maps are tessellated (step 3) into nonoverlapping square tiles of the size $30 \times 30$ CCI-LC pixels $(9 \mathrm{~km} \times 9 \mathrm{~km})$. This corresponds to $0.08^{\circ} \times 0.08^{\circ}$ at the equator. The size of a tile is a free parameter which determines the scale on which the change of landscape is assessed. Given the resolution of CCI-LC our choice of tile's size corresponds to a mesoscale, it's small enough to provide a high resolution on the global scale but large enough for tiles to encompass meaningful landscapes.

\subsection{Magnitude of landscape change}

We calculate (step 4) a dissimilarity value between 1992 mosaic and 2015 mosaic in each tile. Quantitative assessment of dissimilarity between two landscape mosaics requires a mathematical representation of the mosaic and a definition of a dissimilarity function.

In our approach, the mosaic (pattern of land cover categories) in a landscape is mathematically described 


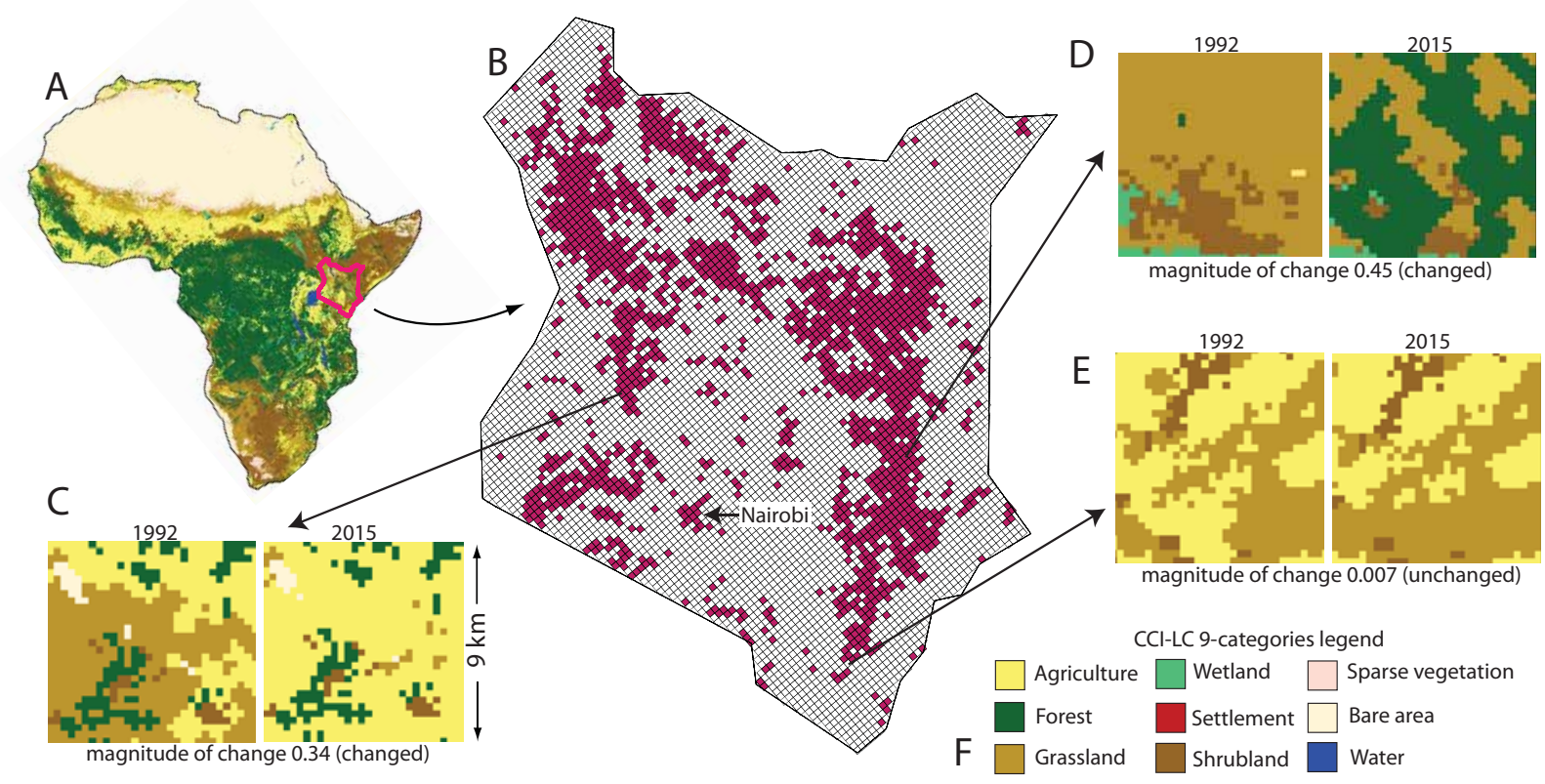

Figure 2: Methodology of change detection shown using the country of Kenya as an example. (A) 2015 CCI-LC map of Africa, the red outline indicates the location of Kenya. (B). The area of Kenya divided into a grid of $9 \mathrm{~km} \times 9 \mathrm{~km}$ tiles, red tiles indicate where landscape mosaic has changed during the 1992-2015 period. (C)-(D) Examples of changed tiles. (E) An example of an unchanged tile. (F) Legend of 9 IPCC land cover categories.

by a normalized histogram (the sum of all its bins equals to 1) of co-occurrence pattern features (Barnsley and Barr, 1996; Chang and Krumm, 1999). Briefly, pattern features are the pairs of land cover categories assigned to two neighboring pixels. Histogram counts and bins the features from eight co-occurrence matrices calculated for eight different displacement vectors along the eight principal directions (see Niesterowicz et al. (2016) for an illustrative example). The result is a histogram with $\left(N^{2}+N\right) / 2$ bins, where $N$ is the number of land cover categories; for 9-categories the histogram has 45 bins. Such histogram describes (indirectly but effectively, see Niesterowicz and Stepinski (2016)) composition as well as the spatial configuration of land cover categories within a tile and thus the local landscape.

We use the Jensen-Shannon Divergence (JSD) (Lin, 1991) as a measure of dissimilarity between two mosaics (one for $t_{1}$ and another for $t_{2}$ within the same tile) represented by corresponding normalized histograms $M_{t_{1}}$ and $M_{t_{2}}$. The JSD expresses the informational distance between the two histograms as a deviation between Shannon's entropy of the conjugate of the two histograms $\left(M_{t_{1}}+M_{t_{2}}\right) / 2$ and the mean entropy of individual histograms $M_{t_{1}}$ and $M_{t_{2}}$. The value of JSD, de- noted by $d\left(M_{t_{1}}, M_{t_{2}}\right)$, is given by the following formula:

$$
\begin{aligned}
d\left(M_{t_{1}}, M_{t_{2}}\right)= & H\left(\frac{M_{t_{1}}+M_{t_{2}}}{2}\right) \\
& -\frac{H\left(M_{t_{1}}\right)+H\left(M_{t_{2}}\right)}{2}
\end{aligned}
$$

where $H(M)$ indicates a value of the Shannon's entropy of the histogram $M$ :

$$
H(M)=-\sum_{i=1}^{|N|} m_{i} \log _{2} m_{i} .
$$

where $m_{i}$ is the value of $\mathrm{i} t h$ bin in the histogram $M$ and $|N|$ is the number of bins (the same for both histograms). For normalized histograms, the JSD dissimilarity always takes values from 0 to 1 with the value of 0 indicating that two mosaics have identical histograms, and the value of 1 indicating maximum dissimilarity (none of the land cover categories found in one mosaic can be found in the other). The dissimilarity value is used as a quantitative assessment of the magnitude of change, the larger the dissimilarity the bigger the change.

For a landscape with $d\left(M_{t_{1}}, M_{t_{2}}\right)<d_{t h}$, where $d_{t h}$ is a similarity threshold, the landscape change is negligible 
and the landscape may be considered as "unchanged." To divide the tiles in our database into "changed" and "unchanged" we determined (step 5) the value of $d_{t h}$ by selecting a random, stratified sample of 1000 tiles. We labeled these tiles either as changed or unchanged based on visual inspection of tiles' mosaics at $t_{1}$ and $t_{2}$. Applying a decision tree algorithm to a dataset consisting of $\left(d\left(M_{t_{1}}, M_{t_{2}}\right), \text { label }\right)_{i}, i=1, \ldots, 1000$ yielded $d_{t h}=0.012$.

Fig. 2 illustrates our concept of change detection via calculating the dissimilarity between patterns at $t_{1}$ and $t_{2}$ using the country of Kenya as an example. Fig. 2B shows the outline of Kenya tessellated into $9 \mathrm{~km} \times 9 \mathrm{~km}$ tiles. Examples of landscape mosaics at $t_{1}$ and $t_{2}$ in three tiles are shown in panels C, D, and E of Fig. 2. Two tiles, $\mathrm{C}$ and $\mathrm{D}$, labeled as changed, clearly exhibit a change in landscape mosaic, while the tile E, labeled as unchanged, shows no change in its mosaic even so a small number of pixels had transitioned. Red-colored tiles in Fig. 2B indicate areas where landscape mosaic has changed between $t_{1}$ and $t_{2}$ (where $d\left(M_{t_{1}}, M_{t_{2}}\right)>d_{t h}$ ). Note that landscape mosaics in the majority of Kenya's territory had not changed between $t_{1}$ and $t_{2}$. Globally, of $1,640,016$ tiles, only 363,137 (22\%) had changed their landscape mosaics during this period under our definition.

\subsection{Change characterization}

Only changed tiles are subject to change characterization. The remaining portion of the landmass also experienced some change but on the much smaller spatial scale leaving $9 \mathrm{~km}$-scale landscapes unchanged. Change characterization aims at an explicit description of the change. Descriptors of change are calculated and saved as tiles' attributes (step 6 in Fig. 1).

Bins of a histogram of from-to transitions aggregated from 900 pixels within a tile make up the first set of change descriptors. With 9 land categories there are potentially 72 types of transitions, but since no pixel with the "settlement" category changed to another category during the $\Delta t$ period (see section 3 ) there are actually only 64 types of transitions. For each tile we save a histogram with 65 bins (most of them equal to 0 ), the additional bin is a share of 900 pixels that did not change their category. The bins are normalized to shares so they all sum to 1 . These descriptors provide the most detailed and direct information on change within a tile. Gross and net changes to coverages of land cover categories can be calculated using these descriptors. In addition, because the information is stored in the database, analysts may use these descriptors to SQL-search for geo- graphic locations where specific transitions occurred at a specified range of intensities.

To enable checking our database versus the earlier results by Li et al. (2018) we calculated the second set of descriptors consisting of a composition of PFTs in each tile at $t_{1}$ and $t_{2}$. Composition of PFTs in each pixel is calculated using a cross-walking table (Poulter et al., 2015) between 22 CCI-LC classes and 13 PFTs (broadleaf evergreen tree, broadleave deciduous tree, needleleaf evergreen tree, needleleave deciduous tree, broadleaf evergreen shrub, broadleave deciduous shrub, needleleaf evergreen shrub, needleleave deciduous shrub, natural grass, managed grass, bare soil, water, snow/ice). Aggregation of PFTs compositions from 900 constituting pixels gives their composition in the entire tile.

Histograms of transitions provide in-depth information about the land change but it is ill-suited for the visualization of the global change. The goal of such visualization is to give a simple, single map overview of what and where has changed during the $1992-2015$ period. A map of 64 types of from-to at-pixel transitions does not fulfill such goal by being too busy both thematically as well as spatially thus leading to a salt-and-pepper effect which distracts from observing the broad-scale trends. In order to obtain an informative broad-scale visualization of change we classified 64 transitions into just 13 "change trends": cropland gain, cropland loss, forest gain, forest loss, grassland gain, grassland loss, shrubland gain, shrubland loss, wetland gain, wetland loss, urban gain, water gain, and water loss (see Table 1). Note that in this classification we do not distinguish between sparse and bare categories. Reclassification of transitions into change trends causes information loss as multiple transitions are reclassified to a single trajectory. The labels of trajectories reflect our assumed priority. For example, an agriculture $\rightarrow$ grassland transition is classified as "cropland loss" but it could also be classified as "grass gain."

Bins of a histogram of change trends aggregated from 900 pixels within a tile make up the third set of change characterization. For each tile we save 14 bins, the additional bin is a share of stable pixels. The values are normalized to shares so they all sum to 1 . For $77 \%$ of the tiles, a single trend type completely dominates (on average $96 \%$ of changed pixels in the tile are assigned to a dominant change trend). For the remaining $23 \%$ of tiles on average, $63 \%$ of changed pixels are assigned to a dominant trend. Thus, a trend change for the entire tile can be inherited from the dominant trend of its constituent pixels which makes possible mapping change at the resolution of $9 \mathrm{~km}$ instead of $300 \mathrm{~m}-\mathrm{a}$ desirable 
Table 1: Reclassification of land cover transitions into landscape trend change types

to 2015

\begin{tabular}{|c|c|c|c|c|c|c|c|c|c|}
\hline & Agriculture & Forest & Grass & Wetland & Settlement & Shrub & Sparse & Bare & Water \\
\hline Agriculture & Stable & Forest $\uparrow$ & Crop $\downarrow$ & Wetland $\uparrow$ & Urban $\uparrow$ & Shrub $\uparrow$ & $\overline{\text { Crop } \downarrow}$ & Crop $\downarrow$ & Water $\uparrow$ \\
\hline Forest & Forest $\downarrow$ & Stable & Forest $\downarrow$ & Forest $\downarrow$ & Urban $\uparrow$ & Forest $\downarrow$ & Forest $\downarrow$ & Forest $\downarrow$ & Water $\uparrow$ \\
\hline Grass & Crop $\uparrow$ & Forest $\uparrow$ & Stable & Wetland $\uparrow$ & Urban $\uparrow$ & Shrub $\uparrow$ & Grass $\downarrow$ & Grass $\downarrow$ & Water $\uparrow$ \\
\hline Wetland & Wetland $\downarrow$ & Forest $\uparrow$ & Wetland $\downarrow$ & Stable & Urban $\uparrow$ & Wetland $\downarrow$ & Wetland $\downarrow$ & Wetland $\downarrow$ & Water $\uparrow$ \\
\hline Settlement & Urban $\downarrow$ & Urban $\downarrow$ & Urban $\downarrow$ & Urban $\downarrow$ & Stable & Urban $\downarrow$ & Urban $\downarrow$ & Urban $\downarrow$ & Urban $\downarrow$ \\
\hline Shrub & Crop $\uparrow$ & Forest $\uparrow$ & Grass $\uparrow$ & Wetland $\uparrow$ & Urban $\uparrow$ & Stable & Shrub $\downarrow$ & Shrub $\downarrow$ & Water $\uparrow$ \\
\hline Bare & Crop $\uparrow$ & Forest $\uparrow$ & Grass $\uparrow$ & Wetland $\uparrow$ & Urban $\uparrow$ & Shrub $\uparrow$ & Stable & Stable & Water $\uparrow$ \\
\hline Water & Water $\downarrow$ & Water $\downarrow$ & Water $\downarrow$ & Water $\downarrow$ & Urban $\uparrow$ & Water $\downarrow$ & Water $\downarrow$ & Water $\downarrow$ & Stable \\
\hline
\end{tabular}

$\uparrow$ indicates gain, $\downarrow$ indicates loss, italic font indicates trends not observed in the 1992-2015 set of transitions

property from the point of view of getting a lucid visualization.

For a global thematic map of landscape change, each tile is assigned a color on the basis of its change trend and the percentage of changed pixels. Tiles where this percentage is $>30 \%$ are classified as "large change", those where this percentage is between $10 \%$ and $30 \%$ are classified as "medium change", and those where this percentage is below $10 \%$ are classified as "small change." Altogether, the map has 39 categories, each a combination of change trend, which describes a type of change, and percentage of tile's area that had changed, which indicates an intensity of change.

The entire database of landscape change is available for download from http://sil.uc.edu. The distributed database is projected back to Lat/Lon coordinates.

\section{Results}

\subsection{Global summary of category transitions}

Fig. 3 summarizes 1992-2015 transitions between the 9 land cover categories calculated using our database. The lower row of pie-diagrams pertains to gross losses in land cover categories. The red numbers below pie-diagrams indicate total loss of an area (in $\mathrm{km}^{2}$ ) in a given land cover category to other land cover categories. A pie diagram illustrates a percentage breakup of this loss going to other categories. For example, during the $\Delta t$ period $799,942 \mathrm{~km}^{2}$ was lost from the agriculture. The pie diagram indicates that most of this loss was to the forest $(60 \%)$ and the settlement $(28 \%)$. The upper row of pie-diagrams pertains to global gross gains in land cover categories. The green numbers above pie-diagrams indicate the total gain in an area (in $\mathrm{km}^{2}$ ) of a given land cover category from other land cover categories. A pie diagram illustrates a percentage breakup of this gain coming from other categories. For example, during the $\Delta t$ period 1,558,080 $\mathrm{km}^{2}$ was gained by the agriculture. The pie diagram indicates that most of this gain was from the forest (60\%), shrubland $(16 \%)$, grassland $(11 \%)$, and sparse $(10 \%)$. Transitions larger than $10 \%$ of category loss are illustrated by lines connecting the loss pie-diagrams with gain pie-diagrams; colors of the lines indicate the destination and the widths are proportional to the percentage of the loss going to a given destination. The blue numbers indicate the net change in an area (in $\mathrm{km}^{2}$ ) of a given land cover category; for example, a net change in an area covered by agriculture was $+758,138 \mathrm{~km}^{2}$.

\subsection{Global changes in terms of PFTs}

Table 2 shows global gross and net changes to major PFTs calculated using our database. We have grouped all types of trees and all types of shrubs into "trees" and "shrubs" types. We also have grouped bare soil, water, and snow/ice into one non-vegetated type. This table has two sections, the upper section pertains to "changed" landscapes defined in section 2.2, and the lower section pertains to the entire landmass which also include "unchanged" landscapes which, however, may contribute to changes in PFTs. For each type, we list areas covered in 1992 and 2015, the gross area lost, the gross area gained, and the net change.

The first observation is that changes to the coverage of PFTs occurred, as expected, mainly within the "changed" zone. A percentage of change within this zone, $(\text { area lost }+ \text { area gained })_{\text {zone }} /(\text { area lost }+ \text { area gained })_{\text {all }}$, is $82 \%$ for trees, $82 \%$ for shrub, $80 \%$ for natural grass, and $84 \%$ for managed grass, and $82 \%$ for non-vegetated. A percentage of net change within this zone is $83 \%$ for trees, $88 \%$ for shrub, $71 \%$ for natural grass, and $95 \%$ for managed grass. 


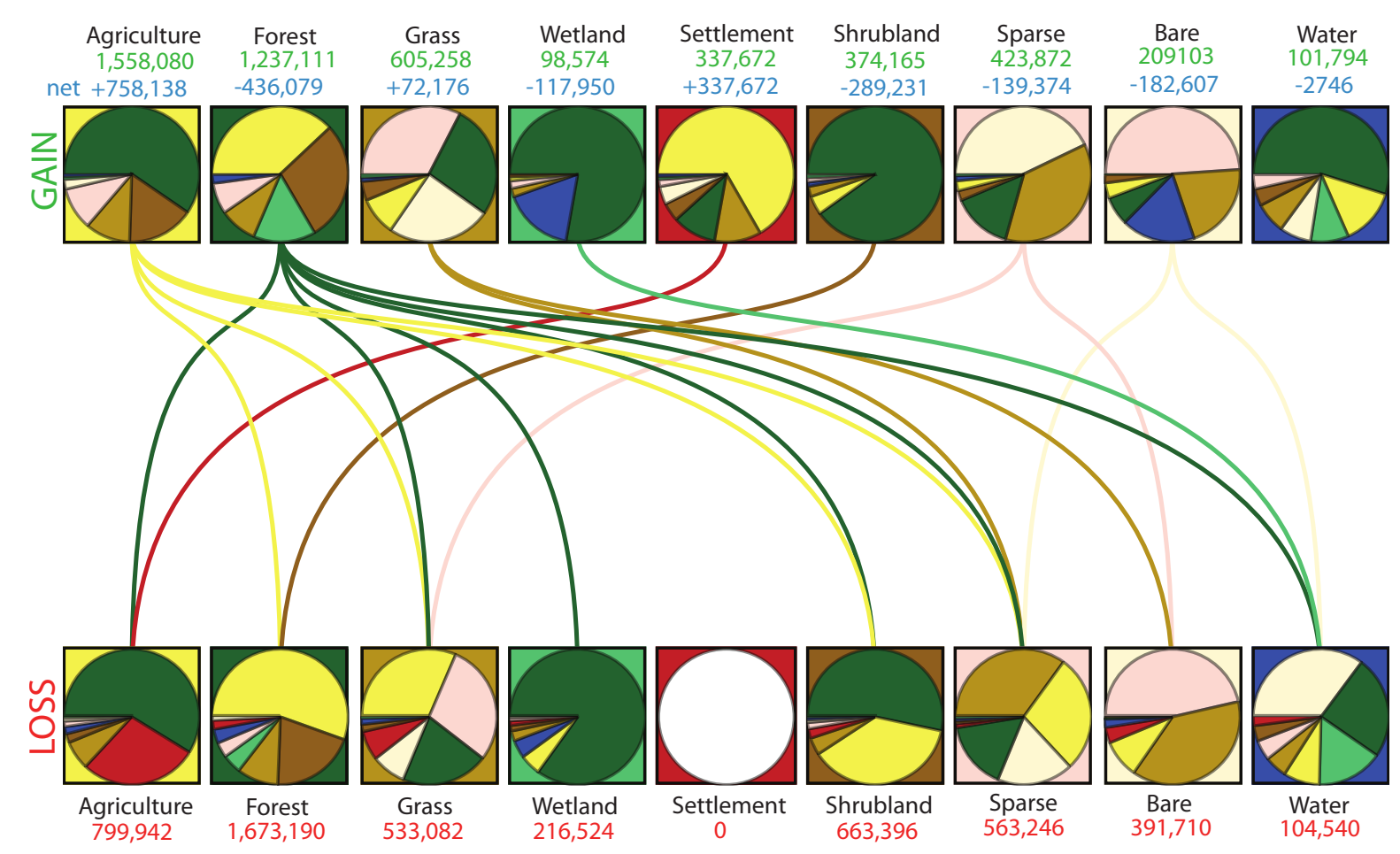

Figure 3: Global summary of major 1992-2015 transitions between the nine CCI-LC categories. See the main text for a detailed description of the diagram. Loss, gain, and net numbers are in $\mathrm{km}^{2}$. For the legend to CCI-LC categories see Fig.2.

The second observation is an amount of difference between the net coverage change of PFTs (the net change line in the upper section of Table 2) and the net change in coverage of corresponding CCI-LC categories (blue line in Fig. 3). The relative difference, $\left[(\text { net change })_{\mathrm{CCI}}-(\text { net change })_{\mathrm{PFT}}\right] /(\text { net change })_{\mathrm{CCI}}$, is $6 \%$ between forest and trees, $14 \%$ between agriculture and managed grass, $20 \%$ between grassland and natural grass, $33 \%$ between shrubland and shrub, and $57 \%$ between (bare + water) and non-vegetated. Thus, estimating the net loss of forest and the net gain of crops from CCI-LC transitions yields values similar to those estimated on the basis of changes to PFTs coverages, but estimating changes to other categories and their corresponding PFTs give significantly different results.

The third observation is about the net-to-gross ratio, NGR $=$ (net change $) /($ area lost + area gained $)$, for different PFTs. NGR is 0.24 for trees, 0.28 from shrub, 0.11 for natural grass, 0.37 for managed grass, and 0.02 for non-vegetated. Thus changes to non-vegetated parts of the landmass are highly balanced; the net change being a small fraction of gains and losses. On the other hand, changes to trees, shrub and, managed grass are unbalanced; the net change is a significant fraction of gains and losses.

\subsection{Global thematic map of change trends}

A portion of the global map of change trends showing the North and South Americas is shown in Fig. 4. Maps of change for the entire world, and separately for all continents are given in Supplement S1. Recall from section 2.2 that only $22 \%$ of local landscapes (tiles) has changed. The fact that this percentage appears to be larger in Fig. 4 is due to graphic rendition; the map needs to be zoomed in to reflect the true size of the changed area. It is also important to stress that only a fraction of an area within a changed tile had changed. In $62 \%$ of changed tiles, less than $10 \%$ of the area had changed; these tiles are labeled as "small change" tiles and are drawn on the map in lightest shades. In $26 \%$ of changed tiles $10 \%-30 \%$ of the area had changed; these tiles are labeled as "medium change" and are drawn on the map in intermediate shades, In only $12 \%$ of changed tiles the area had changed by more than $30 \%$; these tiles are labeled as "large change" and are drawn on the map in darkest shades. 
Table 2: Global gross and net changes in coverages of PFTs

\begin{tabular}{|r|r|r|r|r|r|r|}
\hline & Trees & Shrub & Nat. Grass & Man. Grass & Non-vegetated \\
\hline \multicolumn{7}{|c|}{ In the area defined as “changed” } \\
\hline area in 1992 & $8,496,690$ & $3,917,270$ & $4,916,470$ & $5,772,610$ & $6,315,080$ \\
area in 2015 & $8,034,510$ & $3,722,330$ & $5,003,340$ & $6,421,740$ & $6,236,170$ \\
area lost & $-1,175,726$ & $-420,803$ & $-391,598$ & $-451,803$ & $-702,012$ \\
area gained & $+713,552$ & $+225,864$ & $+478,469$ & $+1,100,935$ & $+623,110$ \\
net change & $\mathbf{- 4 6 2 , 1 7 8}$ & $\mathbf{- 1 9 4 , 9 3 9}$ & $\mathbf{+ 8 6 , 8 7 1}$ & $\mathbf{+ 6 4 9 , 1 3 2}$ & $\mathbf{- 7 8 , 9 0 2}$ \\
\hline \multicolumn{7}{|c|}{ In the entire landmass area } \\
area in 1992 & $33,505,300$ & $16,387,800$ & $19,811,100$ & $21,741,800$ & $52,995,900$ \\
area in 2015 & $32,946,300$ & $16,165,500$ & $19,933,900$ & $22,428,600$ & $52,967,300$ \\
area lost & $-1,437,286$ & $-507,651$ & $-479,499$ & $-588,094$ & $\mathbf{- 8 1 9 , 3 1 1}$ \\
area gained & $+878,162$ & $+285,359$ & $+602,368$ & $+1,274,869$ & $+790,683$ \\
net change & $\mathbf{- 5 5 9 , 1 2 4}$ & $\mathbf{- 2 2 2 , 2 9 2}$ & $\mathbf{+ 1 2 2 , 8 6 9}$ & $\mathbf{+ 6 8 6 , 7 7 5}$ & $\mathbf{- 2 8 , 9 0 2}$ \\
\hline
\end{tabular}

All areas in $\mathrm{km}^{2}$. Areas in 1992 and 2015 rounded to six significant digits.

When interpreting change trend map it is important to keep in mind that it is based on transitions between 9 IPCC categories and not on transitions between original 22 CCI-LC categories. The IPCC $\rightarrow$ CCI-LC correspondence table (ESA, 2017) shows which CCI-LC categories are grouped into a single IPCC category. In particular, four CCI-LC categories corresponding to agriculture are grouped together. One of these categories, "mosaic natural vegetation" contains $<50 \%$ cropland, thus in some cases, land classified in this category may contain a small percentage of an agricultural area. In cases of transition to "mosaic natural vegetation," the change trend map shows "agriculture gain" where in fact only a small gain of an agricultural land cover may have occurred. In addition, eight CCI-LC categories corresponding to the forest are combined into a single IPCC category, thus, for example, "large forest gain" may indicate areal gains of different types of forested landscape, some corresponding to more actual trees than others. The change trend map is intended as the firstlook resource to quickly identify areas of potential interest. When areas of interest are identified they should be verified by overlaying the change trend map with original 1992 and 2015 CCI-LC maps (using our database).

A detailed discussion of the change trend map is beyond the scope of this paper. Briefly, the map shows that largest areas of forest loss are observed in the tropical regions, but forest loss is also observed elsewhere, in particular in Russia, Scandinavia, Baltic countries, and Canada. The top three transitions responsible for forest loss are forest $\rightarrow$ agriculture, forest $\rightarrow$ shrubland, and forest $\rightarrow$ grassland, together responsible for $84 \%$ of the loss. The map also shows significant areas of forest gain, especially in northern Russia, northern Canada, and in Africa. The top three transitions responsible for forest gain are agriculture $\rightarrow$ forest, shrubland $\rightarrow$ forest, and wetland $\rightarrow$ forest, together responsible for $75 \%$ of the gain.

The prominent areas of crop expansion on the change trend map are in northern Kazakhstan, southern Russia, northern Iran, the Sahel region in Africa, northern Algeria, northeastern China, and New South Wales in Australia. Check with original CCI-LC 1992 and 2015 maps confirms those findings except in Kazakhstan and Sahel, where transitions are predominantly to "mosaic natural vegetation" which pertain to only small agricultural gains (see above). The top three transitions responsible for crop gain are shrubland $\rightarrow$ agriculture, grassland $\rightarrow$ agriculture, and sparse vegetation $\rightarrow$ agriculture, together responsible for $84 \%$ of the gain. The growth of urban areas is around preexisting large cities but a more widespread growth around smaller cities is also observed in eastern China, northern India, and parts of Europe. The top three transitions responsible for urban gain are agriculture $\rightarrow$ urban, grassland $\rightarrow$ urban, and forest $\rightarrow$ urban, together responsible for $76 \%$ of the gain. Loss of wetlands is a prominent feature along the Atlantic and Gulf coasts of the United States. The top two transitions responsible for wetland loss are wetland $\rightarrow$ agriculture and wetland $\rightarrow$ grass, together responsible for $68 \%$ of the loss. The most prominent feature of water loss is a disappearance of the Aral Sea. Prominent water gain is observed along the existing Amazonian River network. Note that this is a low magnitude gain, we speculate that it may be due to interannual fluctuations of water level and/or clearing of river banks.

\section{Discussion and conclusions}

The release of ESA CCI-LC dataset opened a possibility to study land change on the global scale over a pe- 


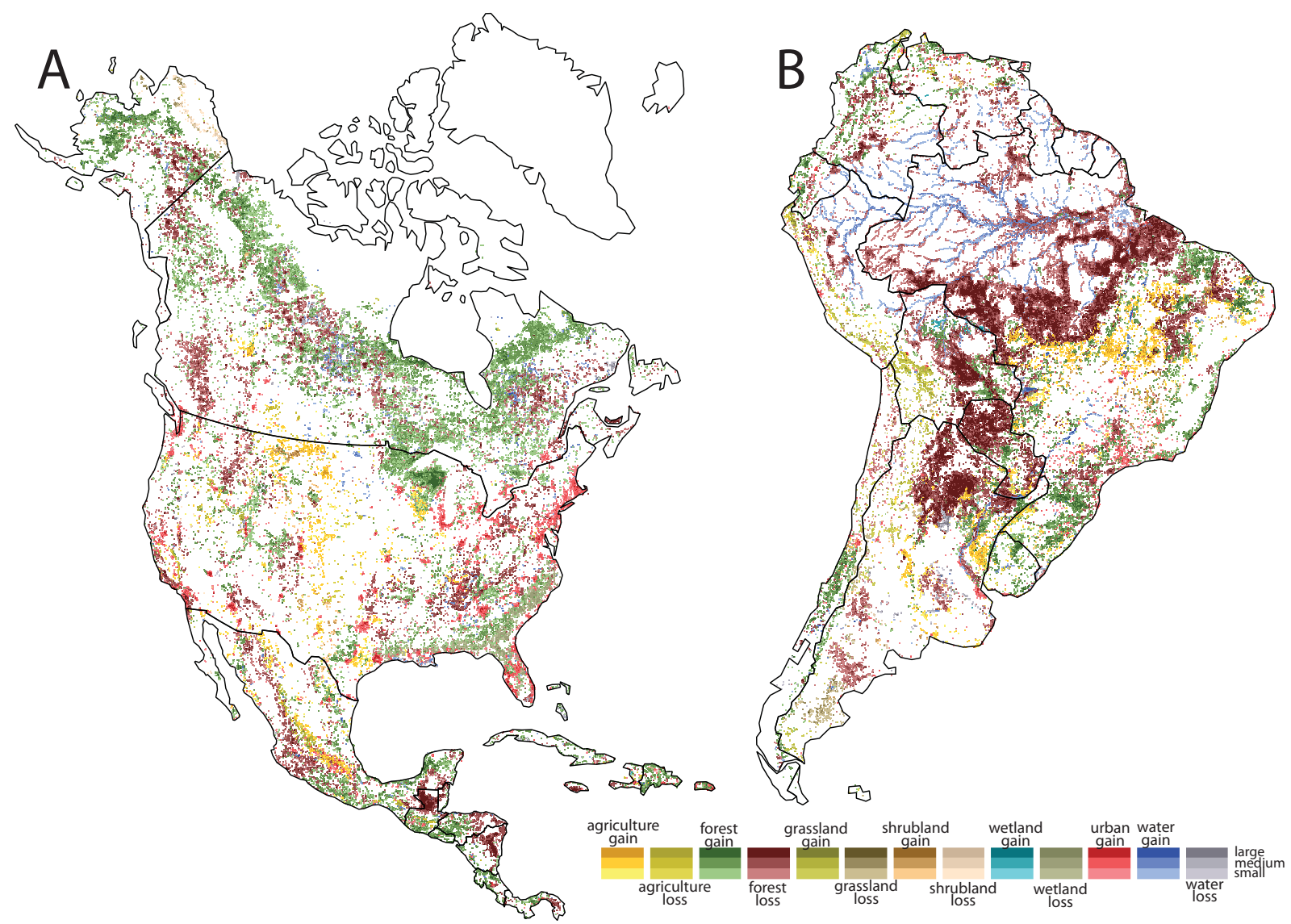

Figure 4: Map of 1992-2015 landscape change trajectories for North America (A) and South America (B). Local landscapes are colored depending on their change trajectories and a percentage of changed area; small $<10 \%$, medium $(10 \%$ to $30 \%)$, and large $(>30 \%)$.

riod of almost a quarter of a century. Land changes are of interest for multiple disciplines. The CCI-LC team is focused on changes to the land cover from the point of view of modeling the climate change. Their recent CCI-LC-based assessment (Li et al., 2018) of gross and net land cover changes reflects this interest by focusing on PFTs and by aggregating data to the resolution of typical LSM.

In this paper, we described a creation of CCI-LCbased spatial database to be used for the assessment and visualization of land change from the point of view of landscape ecology and environmental studies. Different focus calls for a different approach. Table 3 summarizes main differences between the approach presented in this paper and the one taken by $\mathrm{Li}$ et al. (2018). The most prominent difference is the size of aggregation units, our units are smaller and their sizes and shapes are all the same. The size reflects our interest in mesoscale landscapes. Equal size and shape of all landscapes en- able direct comparison between their patterns. Another difference is our interest in quantitative assessment of the magnitude of landscape's pattern change. A map of change magnitude enables identification of hot and cold spots of land change over the entire landmass either visually or by the means of SQL-search.

Also, our perspective is GIS-centric and map-centric. This is reflected in the products associated with this paper. The vector database is intended to be queried using a large number of attributes associated with each landscape. The available attributes pertain to landscape composition in 1992 and 2015, as well as to the characteristics of change. The thematic map of change offers an information-rich (location, change magnitude, and change type) visualization of 19922015 land change in a single map. This distinguishes it from the FAO (Food and Agriculture Organization of the United Nations) web application (http://www.fao.org/faostat/en/\#data/LC) for visualiza- 
Table 3: Comparison of approaches used in this study and in Li et al. (2018)

\begin{tabular}{|l|l|l|l|l|l|l|l|l|}
\hline Study & CCI-LC & Cats. & Agg. units & $\begin{array}{l}\text { Cross-walking } \\
\text { table }\end{array}$ & $\begin{array}{l}\text { Change } \\
\text { mag. }\end{array}$ & $\begin{array}{l}\text { Change } \\
\text { map }\end{array}$ & Comparison & Products \\
\hline $\begin{array}{l}\text { Li et al. } \\
\text { (2018) }\end{array}$ & $\begin{array}{l}\text { entire time } \\
\text { series }\end{array}$ & 37 & $\begin{array}{l}0.5^{\circ} \times 0.5^{\circ} \\
\text { Lat/Lon }\end{array}$ & Li et al. (2018) & no & no & $\begin{array}{l}\text { yes, to four } \\
\text { other datasets }\end{array}$ & annual PFT maps \\
$\begin{array}{l}\text { This } \\
\text { study }\end{array}$ & $1992 / 2015$ & 9,22 & $\begin{array}{l}9 \mathrm{~km} \times 9 \mathrm{~km} \\
\text { Fuller }\end{array}$ & $\begin{array}{l}\text { Poulter et al. } \\
(2015)\end{array}$ & JSD & yes Li & $\begin{array}{l}\text { database of 1992- } \\
\text { et al. (2018) } \\
\text { land change } \\
\text { and thematic map of } \\
\text { landscape change }\end{array}$ \\
\hline
\end{tabular}

tion of the CCI-LC dataset which shows 1992-2015 changes of coverage of individual land cover categories on a country-by-country basis. The design of our thematic map of change required making some arbitrary assumptions (see Table 1 and its description in section 2.3), but, in our judgment, these assumptions are justified because the purpose of the map is only to provide a quick guide to the magnitude and type of 1992-2015 land change. Once areas of interest are spotted on the change map, the unmodified change data is available from the database. The thematic change map has also high educational value as it conveys comprehensive information about the land change in the format accessible to a broad audience.

Our landscape-based approach led to the conclusion that only $22 \%$ of the global area experienced significant land change during the 1992-2015 period on the scale of $81 \mathrm{~km}^{2}$ (an area of our local landscape). In the remaining $78 \%$ of the total landmass the change, if present, is distributed in tracts of land $\ll 81 \mathrm{~km}^{2}$ surrounded by large areas of unchanged land, so its ecological significance is small. Using PFTs, which were calculated not only for the "changed zone" but also for the entire landmass (see Table 2), we demonstrated (section 3.2 ) that the "changed zone" accounts for $\sim 80 \%$ of all change despite occupying only $22 \%$ of the global area. By comparing the net changes of various land cover categories (Fig. 3) and corresponding PFTs (Table 2) in the "changed zone" we have found agreement for forest/trees and agriculture/managed grass, but less of an agreement between different pairs of land cover categories and PFTs.

An inclusion of PFTs in our database provide means of comparison to our results to those of Li et al. (2018). Table 3 underscores differences between ours and $\mathrm{Li}$ et al. (2018) estimates of PFTs; they used a newer version of the cross-walking table and applied it to 37 level 2 land cover categories, whereas we used older crosswalking table (Poulter et al., 2015) and applied it to 22 level 1 land cover categories. Because both studies use
Table 4: Comparison of gross gains and losses and net 1992-2015 changes in PFTs coverages

\begin{tabular}{|r|r|r|r|}
\hline & Trees & Man. Grass & Nat. Grass \\
\hline \multicolumn{4}{|c|}{ Total area } \\
\hline Li et al. (2018) $^{a}$ & 30.4 & 19.3 & 35.7 \\
This study $^{b}$ & 32.9 & 22.4 & 19.9 \\
\hline \multicolumn{4}{|c|}{ Gross gain } \\
\hline Li et al. (2018) & 0.91 & 1.2 & 1.1 \\
This study & 0.88 & 1.3 & 0.6 \\
\hline \multicolumn{5}{|c|}{ Gross loss } \\
\hline Li et al. (2018) & 1.5 & 0.56 & 0.98 \\
This study & 1.44 & 0.59 & 0.48 \\
\hline \multicolumn{5}{|c|}{ Net change } \\
\hline Li et al. (2018) & -0.59 & 0.64 & 0.12 \\
This study & -0.56 & 0.69 & 0.12 \\
\hline
\end{tabular}

All areas in millions of $\mathrm{km}^{2} .{ }^{a} 2000$ data. ${ }^{b} 2015$ data.

the same land cover dataset, the differences in estimates of globally integrated gross and net land changes should only reflect differences in calculating PFTs.

Table 4 shows a comparison between estimates of globally integrated gains and losses as well as 19922015 net changes in coverages of three main PFTs as reported by $\mathrm{Li}$ et al. (2018) and as presented in this study. The top section of Table 4 refers to the total area and is reported at different years, so a direct comparison is not possible, however, the difference in natural grass coverage stands out as especially large. For trees (forest) and managed grass (cropland), both studies report comparable gains, losses, and net changes. For natural grass (grassland) the gains and losses reported by (Li et al., 2018) are twice as large as calculated by us, but the net change is the same. An agreement for forest and cropland and the discrepancy for grassland are attributed directly to the use of different cross-walking tables. Comparing the two tables it is clear that conversion factors for the natural grass used by $\mathrm{Li}$ et al. (2018) have increased significantly for many land cover categories from their values reported in Poulter et al. (2015), whereas conversion factors for trees and crop- 


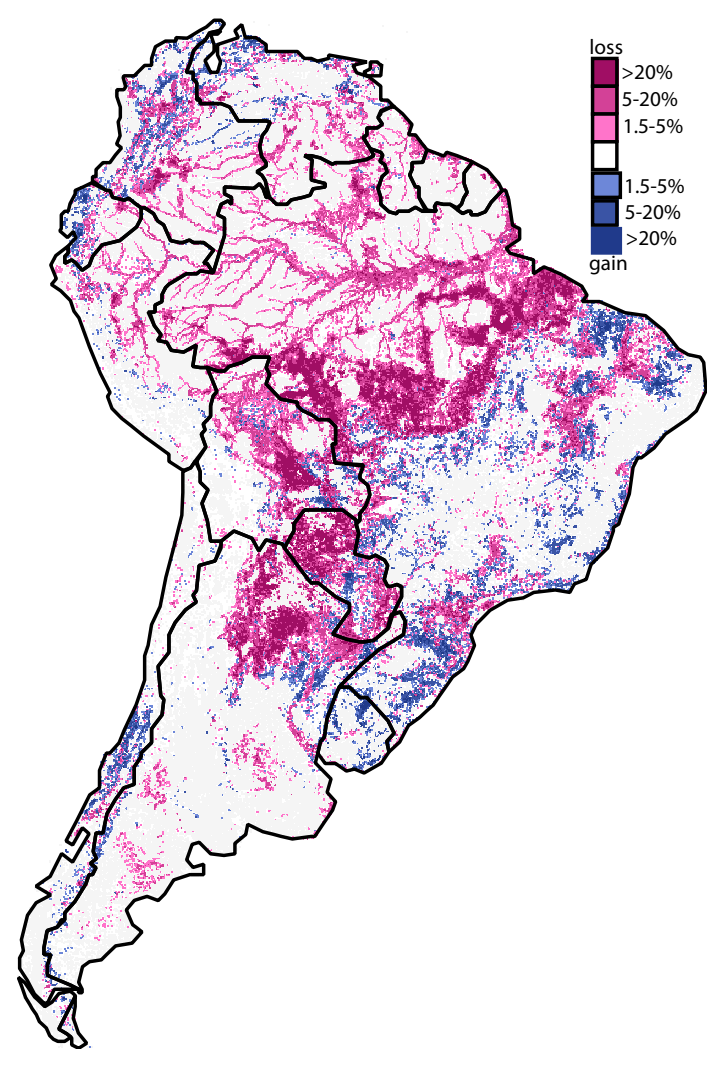

Figure 5: Map of forest losses (red) and gains (blue) in South America during the 1992-2015 period based on our calculation of tree cover. Different shades of red/blue indicate the magnitude of loss/gain in units of percentage of area in a $9 \mathrm{~km} \times 9 \mathrm{~km}$ tile. To be compared with the Hansen's et al. map ( http://www.globalforestwatch.org).

land changed less. The accuracy of PFTs conversion factors is important for LSMs, but it is less important for landscape ecology where land cover transitions are of primary interest. Interestingly, using level 1 categories and older version of conversion table makes little difference for estimates of gross changes for trees and cropland.

Comparison in Table 4 gives a positive check on the expectation that our dataset, when applied to the same questions as were asked in $\mathrm{Li}$ et al. (2018) provides approximately the same answers, and would provide even more similar answers if not for different crosswalking tables used by the two studies. Hence, conclusions drawn by Li et al. (2018) from the comparison of gross and net changes derived from CCI-LC and other datasets (Hurtt et al., 2011; Hansen et al., 2013; Goldewijk et al., 2017; Houghton and Nassikas, 2017) are applicable to our data as well (with a cross-walking table caveat).
In particular, we confirm that using CCI-LC to derive gross changes of forest leads to underestimation of the magnitude of losses and an overestimation of the magnitude of gains in comparison to the reference study by Hansen et al. (2013). This discrepancy stems from the much finer resolution of Hansen et al. (2013) Landsat data and their use of the direct spectral change instead of the post-classification change detection method. The CCI-LC definition of the forest category (at level 1) requires as little as $15 \%$ of $300 \mathrm{~m} \times 300 \mathrm{~m}$ pixel's area to be covered by trees (ESA, 2017) in order to be labeled as "forest." This leads to an underestimation of forest loss using a post-classification method because predominantly forested CCI-LC pixels must experience large forest loss (so their tree cover drops below 15\%) before they are re-labeled to non-forest categories. In other words, statistically, losses of forest in small tracts of land are ignored by the post-classification change detection but detected by the direct comparison of much smaller Landsat pixels. The CCI-LC definition of forest category also leads to an overestimation of forest gain, because pixels labeled as non-forest may transition to the forest category by adding tree cover to just a few percentages of their total area.

Although we have found that using CCI-LC to accurately estimate the magnitude of forest loss/gain may not replace estimates based on high-resolution data and direct spectral change detection (Hansen et al., 2013), the CCI-LC data can be used to illustrate the geography of the forest change. Fig. 5 shows our map of forest losses/gains during the 1992-2015 period in South America. Comparison of this map to the Hansen's et al. map (http://www.globalforestwatch.org) reveals strong similarities in geographical distributions for forest losses and gains, although the gains are more widespread in our map. This geographical correspondence between the two maps extends the entire landmass.

Overall, our global spatial database of 1992-2015 landscape change provides a SQL-searchable, GISbased resource that can be cross-referenced with other global databases, for example, that of terrestrial ecoregions (Olson et al., 2001), while our thematic map of change provides an all-encompassing, global-scale single-map visualization of land change in the 19922015 period. Their mesoscale resolution (less detailed than the CCI-LC but much more detailed than $0.5^{\circ} \times 0.5^{\circ}$ of Li et al. (2018) agglomeration) is well-suited to land change studies on the variety of spatial scales.

Acknowledgments. This work was supported by the University of Cincinnati Space Exploration Institute. 


\section{References}

Alkama, R., Cescatti, A., 2016. Biophysical climate impacts of recent changes in global forest cover. Science, 351(6273), pp.600-604. 351, 600-604.

Barnsley, M. J., Barr, S. L., 1996. Inferring urban land use from satellite sensor images using kernel-based spatial reclassification. Photogrammetric Engineering and Remote Sensing 62 (8), 949-958.

Bonan, G. B., 2008. Forests and climate change: forcings, feedbacks, and the climate benefits of forests. Science 320, 1444-1449.

Chang, P., Krumm, J., 1999. Object recognition with color cooccurrence histograms. In: In Proceedings of IEEE Conference on Computer Vision and Pattern Recognition, Fort Collins, CO, June 2325, 1999. IEEE Computer Society Conference.

Coppin, P., Jonckheere, I., Nackaerts, K., Muys, B., Lambin, E., 2004. Revie Article Digital change detection methods in ecosystem monitoring: A review. International Journal of Remote Sensing 25(9), 1565-1596.

DeFries, R., 2013. Why forest monitoring matters for people and the planet. In: Achard, F., Hansen, M. C. (Eds.), Global forest monitoring from Earth observation. CRC Press/ Taylor \& Francis Group, pp. $1-14$.

DeFries, R. S., Foley, J. A., Asner, G. P., 2004. Land-use choices: Balancing human needs and ecosystem function. Frontiers in Ecology and the Environment 2(5), 249-257.

ESA, 2017. European Space Agency Land Cover CCI Product User Guide Version 2.0. Tech. rep.

Goldewijk, K. K., Beusen, A., Doelman, J., Stehfest, E., 2017. Anthropogenic land use estimates for the HoloceneHYDE 3.2. Earth Syst. Sci. Data 9, 927-953.

Gray, R. W., 1995. Exact transformation equations for Fuller's world map. Cartographica: The International Journal for Geographic Information and Geovisualization 32(3), 17-25.

Hansen, M. C., Potapov, P. V., Moore, R., Hancher, M., Turubanova, S., Tyukavina, A., Thau, D., Stehman, S. V., Goetz, S. J., Loveland, T. R., Kommareddy, A., Egorov, A., Chini, L., Justice, C. O., Townshend, J. R., 2013. High-resolution global maps of 21stcentury forest cover change. Science $342, .850-853$.

Hansen, M. C., Stehman, S. V., Potapov, P. V., 2010. Quantification of global gross forest cover loss. Proceedings of the National Academy of Sciences 107(19), 8650-8655.

Houghton, R. A., Nassikas, A. A., 2017. Global and regional fluxes of carbon from land use and land cover change 18502015. Globa Biogeochemical Cycles 31(3), 456-472.

Hurtt, G. C., Chini, L. P., Frolking, S., Betts, R. A., Feddema, J., Fischer, G., Fisk, J. P., Hibbard, K., Houghton, R. A., Janetos, A., Jones, C. D., 2011. Harmonization of land-use scenarios for the period 15002100: 600 years of global gridded annual land-use transitions, wood harvest, and resulting secondary lands. Climatic change 109(1-2), 117-161.

Hussain, M., Chen, D., Cheng, A., Wei, H., Stanley, D., 2013. Change detection from remotely sensed images: From pixel-based to object-based approaches. ISPRS Journal of Photogrammetry and Remote Sensing 80, 91-106.

Kim, D. H., o. Sexton, J., p. Noojipady, Huang, C., Anand, A., Channan, S., Feng, M., Townshend, J. R., 2014. Global, Landsat-based forest-cover change from 1990 to 2000. Remote Sensing of Environment 155, 178-193.

Li, W., MacBean, N., Ciais, P., Defourny, P., Lamarche, C., Bontemps, S., Houghton, R. A., Peng, S., 2018. Gross and net land cover changes in the main plant functional types derived from the annual ESA CCI land cover maps (19922015). Earth Syst. Sci. Data 10 219-234.

Lin, J., 1991. Divergence measures based on the Shannon entropy. IEEE Transactions on Information Theory 37 (1), 145-151.
Lu, D., Li, G., Moran, E., 2014. Current situation and needs of change detection techniques. International Journal of Image and Data Fusion 5(1), 13-38.

Mahmood, R., Pielke, R. A., Hubbard, K. G., Niyogi, D., Dirmeyer, P. A., McAlpine, C., Carleton, A., Hale, R., Gameda, S., BeltránPrzekurat, A., Baker, B., 2014. Land cover changes and their biogeophysical effects on climate. International Journal of Climatology 34(4), 929-953.

Niesterowicz, J., Stepinski, T. F., 2016. On using landscape metrics for landscape similarity search. Ecological Indicators 64, 20-30.

Niesterowicz, J., Stepinski, T. F., Jasiewicz, J., 2016. Unsupervised regionalization of the conterminous U.S. into hierarchical landscape pattern types. International Journal of Geographical Information Science 30(7), 1450-1468.

Olson, D. M., Dinerstein, E., Wikramanayake, E. D., Burgess, N. D., Powell, G. V. N., Underwood, E. C., D'amico, J. a., Itoua, I., Strand, H. E., Morrison, J. C., Loucks, C. J., Allnutt, T. F., Ricketts, T. H., Kura, Y., Lamoreux, J. F., Wettengel, W. W., Hedao, P., Kassem, K. R., 2001. Terrestrial Ecoregions of the World: A New Map of Life on Earth. BioScience 51 (11), 933.

Omernik, J. M., Griffith, G. E., 2014. Ecoregions of the conterminous united states: evolution of a hierarchical spatial framework. Environmental management 54 (6), 1249-1266.

Poulter, B., MacBean, N., Hartley, A., Khlystova, I., Arino, O., Betts, R., Bontemps, S., Boettcher, M., Brockmann, C., Defourny, P., Hagemann, S., 2015. Plant functional type classification for earth system models: results from the European Space Agency's Land Cover Climate Change Initiative. Geoscientific Model Development 8, 2315-2328.

Radke, R. J., Andra, S., Al-Kofahi, O., Roysam, B., 2005. Image change detection algorithms: A systematic survey. IEEE Transactions on Image Processing 14(3), 294-307.

Riitters, K. H., 2011. Spatial patterns of land cover in the United States: a technical document supporting the Forest Service 2010 RPA Assessment. Tech. Rep. Gen. Tech. Rep. SRS-136, Department of Agriculture Forest Service, Southern Research Station, Asheville, NC.

Tewkesbury, A. P., Comber, A., Tate, N., Lambmand, A., Fisher, P. F., 2015. A critical synthesis of remotely sensed optical image change detection techniques. Remote Sensing of Environment 160, 1-14.

Vitousek, P. M., Mooney, H. A., Lubchenco, J., Melillo, J. M., 1997. Human domination of Earth's ecosystems. Science 277(5325), 494-499.

Warner, T. A., Almutairi, A., Lee, J. Y., 2009. Remote sensing of land cover change. In: Warner, T. A., Nellis, D. M., Foody, G. M. (Eds.), The SAGE Handbook of Remote Sensing. SAGE Publications, pp. 459-472.

Wickham, J. D., Norton, D. J., 1994. Mapping and analyzing landscape patterns. Landscape Ecology 9(1), 7-23 
Supplement 1

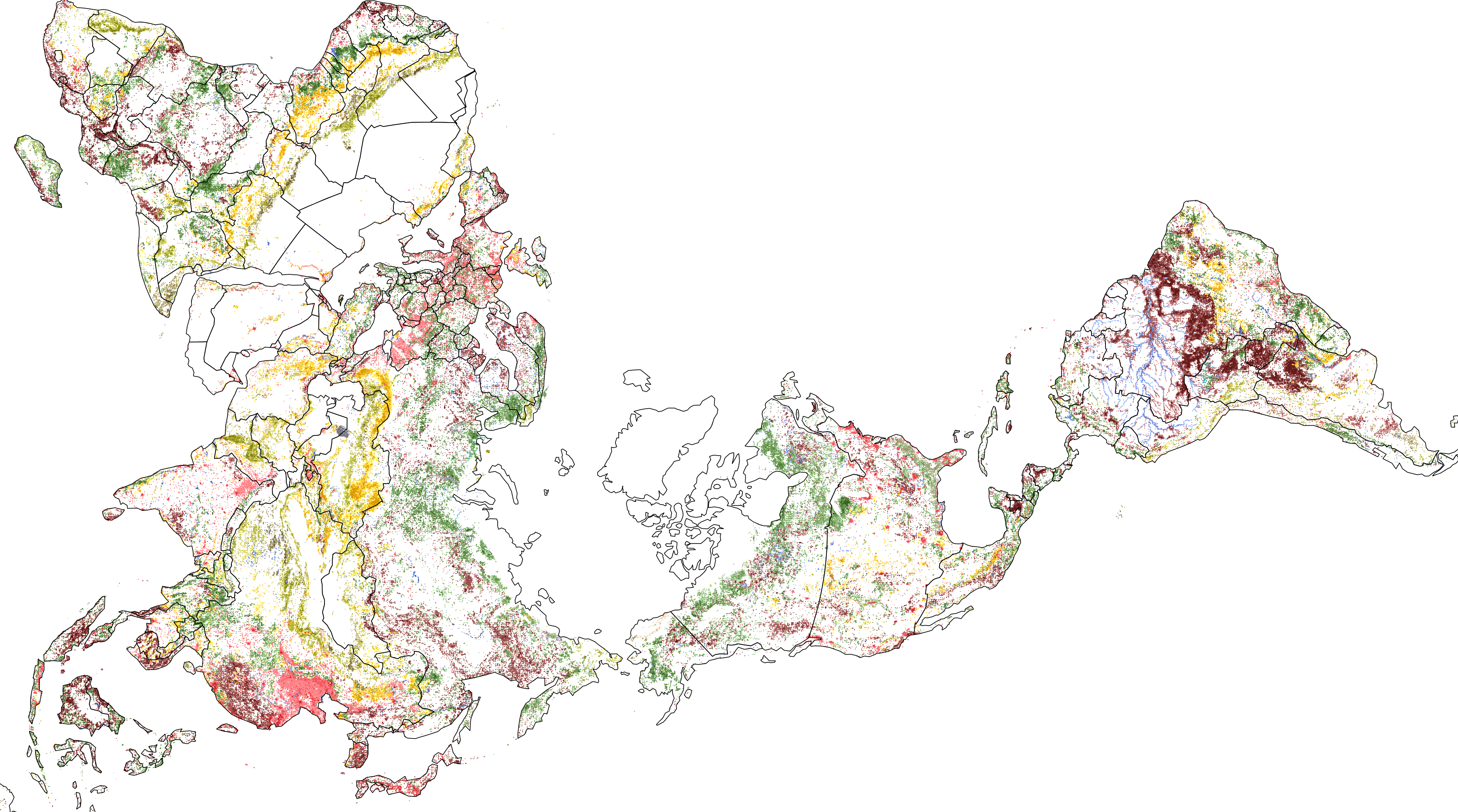

Trajectories of landscape change 1992-2015

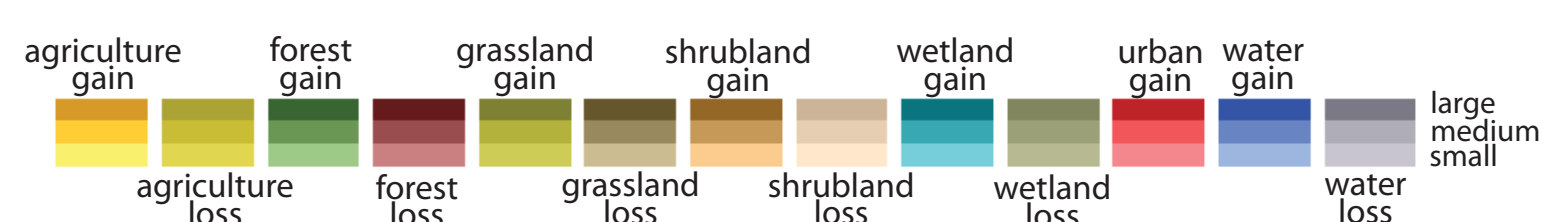

